\title{
Effect of particle shape in grinding mills using a GPU based DEM code.
}

\author{
Nicolin Govender ${ }^{1,2,3}$, Raj Rajamani ${ }^{3}$, Daniel N. Wilke ${ }^{4}$, Chuan-Yu Wu ${ }^{1}$, Johannes Khinast ${ }^{2}$, Benjamin J. Glasser ${ }^{5}$ \\ ${ }^{1}$ Department of Chemical Engineering, University of Surrey, United Kingdom \\ ${ }^{2}$ Research Center Pharmaceutical Engineering, GmbH, Graz, Austria \\ ${ }^{3}$ Department of Metallurgical Engineering, University of Utah, Salt Lake City, USA \\ ${ }^{4}$ Department of Mechanical and Aeronautical Engineering, University of Pretoria, South Africa \\ ${ }^{5}$ Chemical and Biochemical Engineering, Rutgers - The State University of New Jersey, USA
}

\begin{abstract}
The reduction of raw materials into particulate form using grinding mills is an energy and cost intensive task. Optimization of grinding processes is not trivial as obtaining information on the dynamics of media in the mill via experimental means is extremely difficult due to the harsh environment inside, thus computational modeling is the most feasible option to obtain information on the dynamics of the media. However the computational cost of modeling each particle is high, resulting in the shape of the media being approximated by simple shapes and in most cases a reduction in the size of the mill. Even with these simplifications typical simulations take many weeks to months to complete making it unfeasible for quick design prototyping and process optimization. In the last decade the Graphical Processor Unit (GPU) has enabled large scale simulations of tens of millions of spheres in ball mills using the Blaze-DEM GPU code. Recently this code was expanded to provide detailed contact detection for polyhedra using the volume overlap method which is the most accurate approach amongst commercial and academic codes. In this study we firstly validate the code against experiment for polyhedral both as well as spherical particles. We then perform a number of simulations to study the effect of particle shape, in particular angularity and aspect ratio. We clearly demonstrate the importance of accurate particle shape representation in mill simulations by comparing charge profile, power draw and force network for various polyhedra approximations against spheres.
\end{abstract}

\section{Introduction}

Milling Size reduction of granular media is a common task in a large number of industries ranging from the formation of powders in pharmaceutical to the grinding of ore in the minerals processing industry. Owing to the harsh environment when a mill is operation obtaining information on the dynamics of the material inside is very difficult. This lack of information makes the design and optimization of mills a difficult and bespoke process, relying on rule-of-thumb or adhoc design and operation patterns that have not changed in decades. While lab scale experiments can provide some information on the power draw and profile of material inside the mill, information on the forces and stresses that the media is subjected to cannot be readily obtained. Furthermore there is no guarantee that the design characteristics in a lab device scales to an industrial device. Granular material is free form in nature and cannot be described in a closed form solution for more than a few idealized scenarios. Thus direct numerical simulation on the particle scale is required, for which the Discrete Element Method (DEM) is the most popular [1, 2, 3, 4].

Milling Process Autogenous and Semi-autogenous (AG/SAG) mills are universally deployed in many mining operations. The standard practice is a single autogenous mill or semi-autogenous mill followed by a 
pair of ball mills which grinds the run-of-mine ore to a size suitable for flotation. AG/SAG mills produce a product size that is suitable for finish grinding in a ball mill, stirred media mill or a vertical mill. These mills can accomplish the size reduction task of two stages of crushing and screening. Hence, they find numerous applications in the mining industry. Both AG and SAG mills receive ore feed directly from a belt conveyor. Hence, the maximum size of ore is only limited by the conveying capacity of the belt conveyor. The ore size can be as high as $25-35 \mathrm{~cm}$, as one would expect, these naturally occurring ore are random in shape and size $[5,6]$.

In AG mills due to the competency of the ore, larger particles break upon impact, and smaller particles are ground by compressive and attrition forces generated in the tumbling action. In SAG mills, in addition to self-breakage of the ore particles, the grinding balls cause additional breakage. Essentially in these mills which are as large as 9.76 to 12.2 meters in diameter, the grinding action occurs in a system of irregular ore particles and spherical grinding balls, besides impact and attrition grinding also occurs by chipping of sharp edges. Furthermore, weakening of the larger ore particles occurs as internal damage accumulated during repeated impacts. It is well known that notable influences on mill performance include the mill properties such as lifter, liner, grate operating conditions (speed and fill level) and charge characteristics such as particle shape, angularity, aspect ratio.

Although simulations have predominantly focused on spherical particles $[3,5,7]$, particle shape is increasingly being recognized as being important to consider during experimental investigations[8], as well as simulation based investigations. It is also important to take note of some recent studies that aim to quantify particle breakage or particle shape change during mill operations[4]. In addition, ball charge may also deform over time as depicted in Figure 1(d). During milling particles with high angularity (see Figure 1(a) and (c) are worn over time (see Figure 1(b)), due to impacts with other particles and ball charge to be less angular due to high speed impact, abrasion, chipping and nipping of small particles between large particles [4]. As a consequence, particles are often represented as superquadrics [4] that ultimately lack well defined angular edges for particles. However the initial ore feed into a mill has well-defined angular edges as well as newly broken particles inside the milling operation.

Motivation While there have been numerous studies on lifter design power draw, wear and grinding etc, these studies have been limited in the number of particles simulating just spheres or simple superquadrics owing to the large computational cost associated with the DEM. This study intends to quantify the importance of particle shape with defined angular edges experimentally, and demonstrate that polyhedral shape representations are sufficient to capture well defined angular edges. This is achieved by validating our implementation against experiment for cubic and spherical particles at the university of Utah.

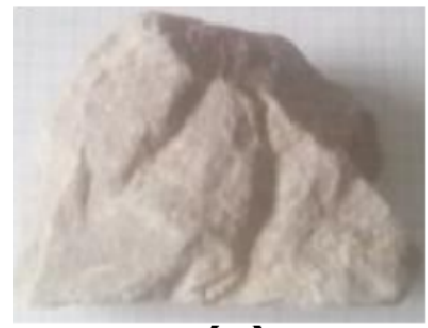

(a)

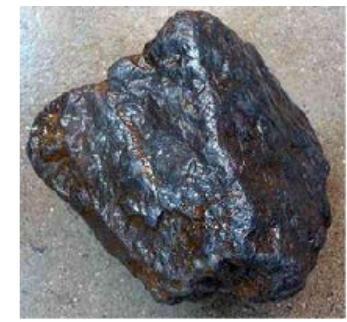

(b)

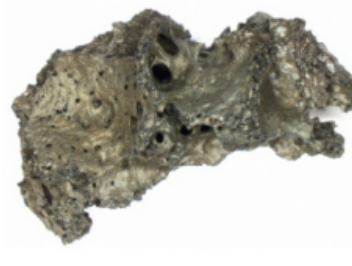

(c)

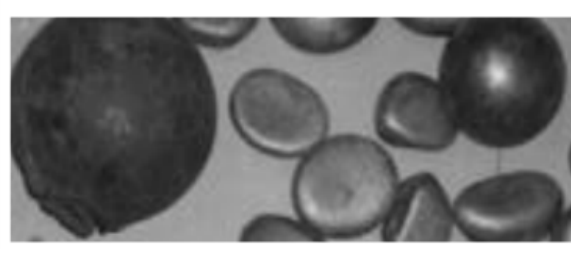

(d)

Figure 1: (a)-(c) Shape of typical material in mills, (d) new and worn balls from a ball mill.

\subsection{Overview of DEM Computing}

Established discrete element software frameworks rely mostly on multi-core central processing unit (CPUs) computing platforms [9] on which the number of polyhedral particles that can be simulated in a realistic time frame on typical workstations is limited. The largest DEM computational simulations to date of polyhedra have been done by the group at the University of Illinois using the code BLOCKS3D [10]. Seung et.al reported in his $\mathrm{PhD}$ thesis [11] that a one second simulation of a million polyhedral particles would require 
18 months wall time. In this thesis an impulse based method similar to what is used in gaming was proposed that require 8.5 days wall time. However such an approach disregards most of the physics that is required for a scientific study and is not considered robust enough for use in engineering applications. Mack et al. simulated 322 polyhedra [12] while Hohner et. al used a few thousand polyhedra particles.

In the last decade the Graphics Processor Unit (GPU) has become increasingly important as an alternative computational platform for discrete element simulations [13, 14]. The GPU has allowed for simulations of tens of millions of particles to be performed within a realistic computing time frame and financial budget $[15,16,6$, $17,18]$. Although the GPU has numerous advantages it also presents various challenges to implementations of the DEM. The complexity of resolving contact for non-trivial particle shapes like polyhedra poses particular challenges on the GPU due to the divergent nature of polyhedral contacts as well as contact models requiring history. This is a result of the limited computational complexity and memory restrictions on the GPU. Although the GPU is an ideal match for DEM simulations the current learning curve associated with GPU development is high as the technology is fairly new and rapidly changing when compared to traditional CPU development. Even with this increased computational capacity majority of the effort has been directed to spherical particles on the GPU $[9,17,18,19]$

\subsection{Overview of BlazeDEM}

The BlazeDEM-GPU framework allows for efficient representation and computation of poly-disperse systems for both spherical and polyhedral (convex and non-convex) shaped particles as depicted in Figure 2.

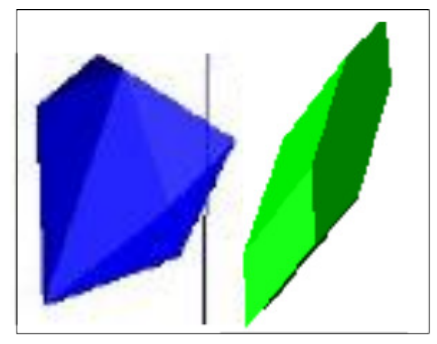

(a)

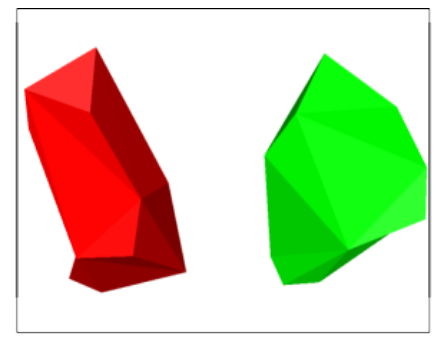

(b)

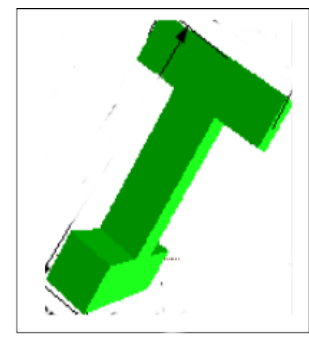

(c)

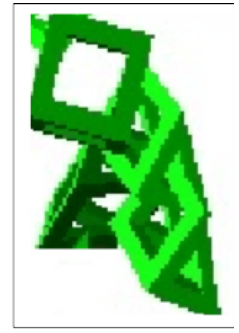

(d)

Figure 2: (a) convex, (b) non-convex, (c) multi convex piece and (d) linked hollow independent convex particle representations in the Blaze-DEM framework.

A key feature of the framework is the geometry can be represented using typical CAD geometry (STL format) or computationally and memory efficient representations called "world" and "volume" objects where possible. "World objects" are planar representations (where any number of vertices can make up a surface) that have two faces joining such that it does not form a convex edge or primitives such as cylinders, cones, etc as opposed to a triangle only STL mesh depicted in Figure 3(a) or a collection of particles as in Figure 3(b) $[19,20,18]$. Finally "volume objects" allow for convex representations with edges and can be considered as polyhedra, which by default do not move under the influence of other particles, however they can deform which allows for surface wear to be captured geometrically. 3(c) depicts the representation in Blaze-DEM of a mill where the surface is a analytical world object (cylinder) and the lifters are "volume objects". A number of industrial devices can be represented using a combination of world and volume objects allowing for memory and computational efficiency. Note: regular STL files can be combined with volume and world objects as depicted in 3(d) where the back end of the mill and pulp lifters are modeled with an STL while the shell and lifters are world and volume objects respectively. 


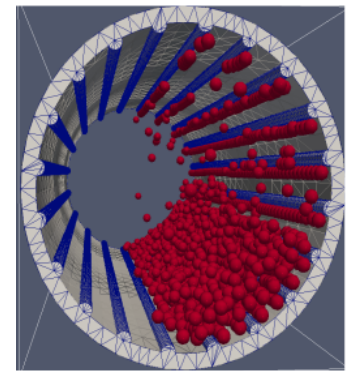

(a)

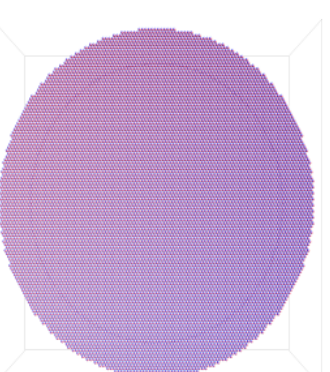

(b)

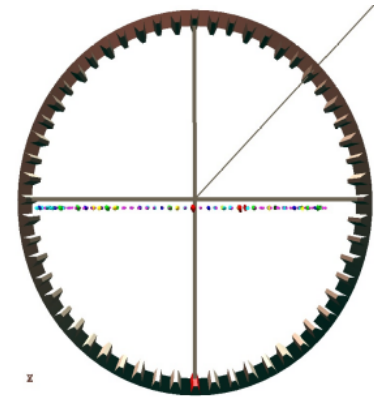

(c)

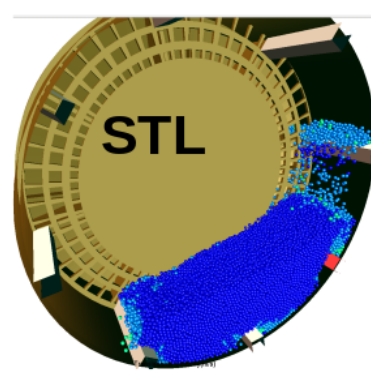

(d)

Figure 3: (a) STL only representation (LIGGGHTS), (b) particle on surface (c) Blaze-DEM analytical (cylinder) and (d) Blaze-DEM planar/STL object geometry representations.

The method used to determine the contact points between a particle and surface is termed as a "ray-tracing approach" $[15,21]$ in BlazeDEM-GPU since it is a parallel method where independent rays ( computational threads ) one from each vertex for polyhedra or the COM (Center Of Mass) for sphere is used to check if there is contact with the surface and return subsequent contact information. While this approach is common practice in computer graphics applications its application to DEM for polyhedra particles to the best of the authors knowledge is not wide spread. Since polyhedra are represented as just a collection of surfaces, the same approach is used [21] to determine the contact points between polyhedra. This approach requires basic algebraic operations and is not iterative like the common-plane approach and hence well suited to the GPU.

The details of all the contact algorithms can be found in [15, 21, 22] here we just provide the modifications for polyhedral particle contact which is relevant to this study as this is computationally the most expensive part of a DEM simulation accounting for as much as $90 \%$ of the total simulation time (polyhedra). Note: BlazeDEM-GPU is able to use multiple GPUs on the same node with an efficiency of $90 \%$ for four GPUs this excellent scaling is due to the use of a novel data transfer algorithm which allows us to hide most of the data transfer costs by overlapping computation with memory transfer.

\section{Contact overview}

In general, contact between particles is first detected using an efficient strategy during the "broad phase" identifying potential contact pairs which are resolved during a computationally more demanding narrow phase to establish whether two particles are actually in contact and if so determine some measure from which the the resulting force direction and magnitude can be computed. The "broad phase" and "narrow phase" for spherical and convex polyhedral particles differ as depicted in Figure 4. The broad phase for spherical and polyhedral shaped particles follow a similar broad phase hashing strategy such as Morton codes. Identified contact particle groups are then directly resolved in a narrow phase for spherical particles to establish contact pairs and ultimately contact measures to calculate force directions and magnitudes. 
SPHERES

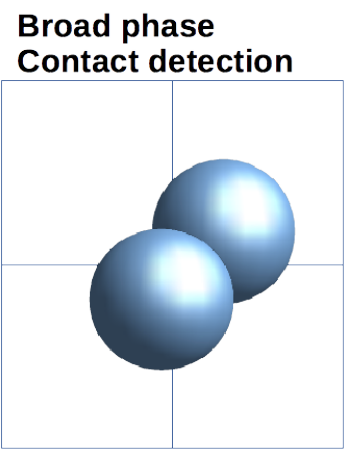

Narrow phase Contact resolution

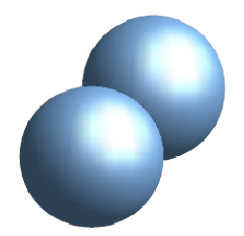

CONVEX POLYHEDRA

\section{Broad phase \\ Contact detection}

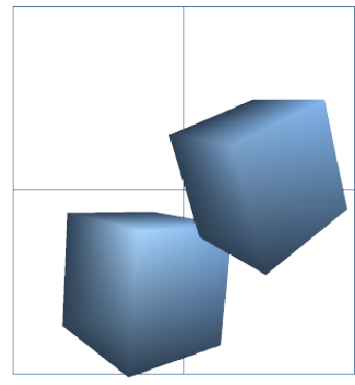

\section{Intermediate phase Contact resolution}

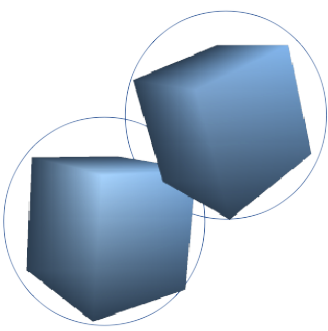

Detail Contact resolution

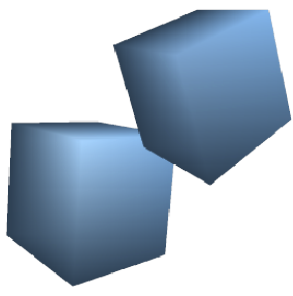

(a)

(b)

Figure 4: Broad phase contact detection and detailed contact resolution for spherical and convex polyhedral particles.

In turn, for convex polyhedral particles the broad phase is followed by an intermediate phase using some bounding primitive to detect contact more accurately than the broad-phase with a much cheaper query than a full contact check as depicted in Figure 4 (b). This is followed by a narrow phase to establish contact pairs and ultimately contact measures to calculate force directions and magnitudes. These forces are estimated in various ways the most popular been the penetration distance approach $[23,24,25]$ where contact is done in various cases such as vertex-face, edge-edge and face-face contact.

Alternatively, the forces can be estimated from the overlapping volume [23, 26, 27], which is the measure we compute exactly in BlazeDEM on GPU architectures. Our current state of the art convex polyhedra contact resolution implementation in BlazeDEM-GPU resolves the overlap volume of two intersecting polyhedra that is significantly harder than finding the overlap distance. The benefit being that it does allow for both the direction and magnitude to be resolved following an energy-conserving contact interaction scheme [28]. We outline the steps involved in computing the overlap volume by considering the intersection of two polyhedral cubes as depicted in Figures 5(a)-(b).

It is important to note that the intersection volume between two intersecting polyhedra is given by the convex hull formed using the vertices at the intersections between the polyhedral edges and surfaces [29]. The first step is to find the intersecting vertices as depicted in Figure 5(a), where after we define the surfaces 
that form the convex hull of the overlap volume. The surface normal $\mathbf{n}_{i}^{A_{j}}$ for each surface $A_{j}$ of the convex hull defined is depicted in Figure 5(b).

To further our understanding consider the contact volume in isolation as depicted in Figure 5(a). The surfaces of this volume is formed by the external surface of one polyhedra that is internal in the other polyhedra. We therefore need to identify which surfaces are internal to which body to ensure that we can compute the associated direction of the reaction force $\mathbf{n}_{i}^{f}$ on particle $i$. Once the surfaces have been affiliated with a respective particle as shown in Figure 5(b), where each color (red and green) is identified with a polyhedral particle. The resultant force is computed by integrating over the surface normals for the contact area as shown by the black lines. The resultant force and surface normals for each particle is respectively depicted in Figures 5(c) and (d).

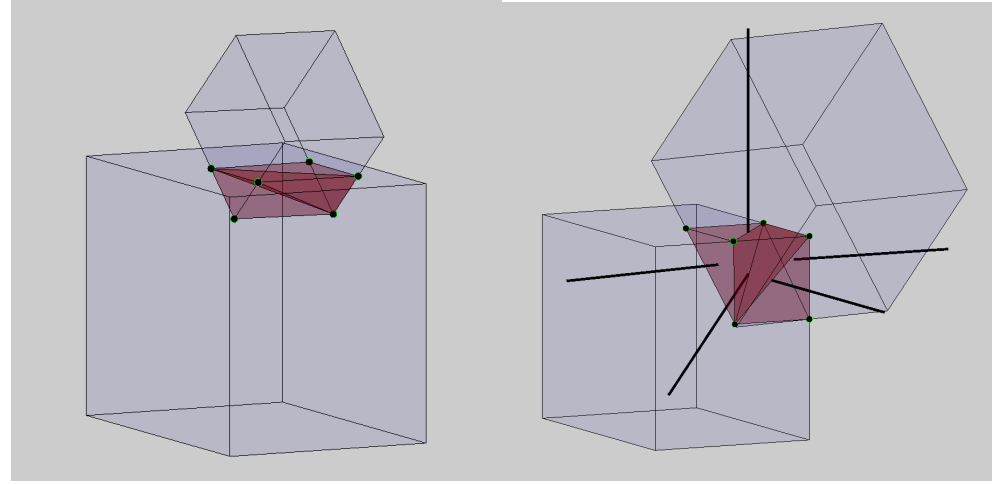

(a)

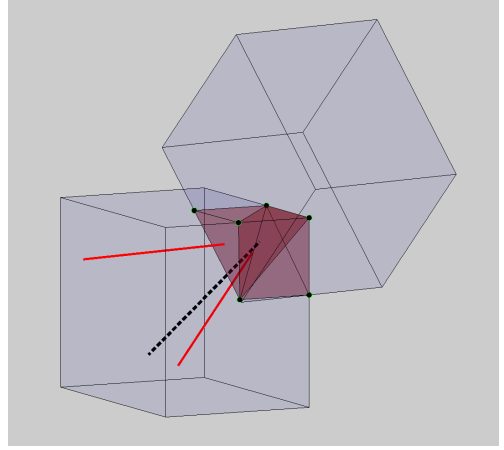

(c) (b)

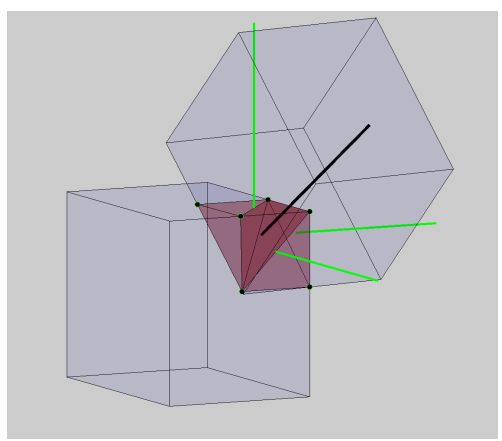

(d)

Figure 5: (a) Overlap between cubes, (b) convex hull with faces, decomposed surfaces (defined by the surface normals) of the contact volume into internal surfaces to the (b) cube on bottom left and the (c) cube on the top right.

Lastly, two additional contact volume properties that needs to be computed the contact Volume $V$ and the COM of the contact volume as it will be required to resolve the elastic contact force. Since, both $V$ and $\mathrm{COM}$ changes between contacts it is required to compute them efficiently on the GPU. The divergence theorem

$$
\iiint_{V}(\nabla \cdot F) V=\oiint S(V) \oiint F \cdot \mathbf{S}
$$

allows us to transform the volume integral of an object with volume $V$ into the surface integral around the boundary surface $S(V)$ of the volume with outward-pointing normal. This can be done by appropriately choosing any vector field $\mathbf{F}\left(x_{1}, x_{2}, x_{3}\right)$ that has a divergence of 1 i.e. $\nabla \cdot \mathbf{F}=1$. This then gives the volume

$$
\oiint S(V) \oiint F \cdot \mathbf{S}=\iiint_{V} V=V
$$

as a surface integral. As an alternative, the contact volume can be computed by breaking the volume into tetrahedra for which efficient closed form expressions exist in computing volumetric and inertial properties 
[30]. Once, these quantities have been computed we calculate the reaction force direction as

$$
\mathbf{n}_{i}^{f}=\frac{\int_{A} \mathbf{n}_{i}^{A} d s}{\left|\int_{A} \mathbf{n}_{i}^{A} d s\right|}=\frac{1}{\sum_{j} A^{j}} \sum_{j} A^{j} \mathbf{n}_{i}^{A_{j}},
$$

which acts on particle $i$ as shown respectively in Figures 5(a) and (b) for the two particles. Here, the direction of the reaction forces are respectively indicated by the dashed and solid black lines. In addition, the computed reaction force acts through the COM of the overlap volume, while the magnitude of the reaction force in turn is proportional to the volume $V$ of the contact volume.

Normal Contact The overlap volume $\Delta V$ and contact normal are resolved exactly for two polyhedral shaped particles in contact as depicted, while for spheres it is just the relative overlap $\delta$. Specifically, a Kelvin-Voigt linear viscoelastic spring dashpot for rigid (hard) particles is considered. This results in an elastic force that stores energy and a dissipative force that dissipates energy given by

$$
\begin{aligned}
\mathbf{F}_{\mathrm{n}}^{\text {spring }} & =\left(\mathrm{K}_{\mathrm{n}} \Delta V^{1 / 3}\right) \mathbf{n}, \\
\mathbf{F}_{\mathrm{n}}^{\text {dash }} & =\mathrm{C}_{\mathrm{n}}\left(\mathbf{V}_{\mathrm{R}} \cdot \mathbf{n}\right) \mathbf{n}
\end{aligned}
$$

where $\mathrm{K}_{n}$ is the spring stiffness $\frac{N}{m}$ (note for spheres $\Delta V^{1 / 3}=\delta$ ), $\mathbf{n}$ the normal direction along which the force acts, $C_{n}$ the damping coefficient $\left(\frac{N s}{m}\right)$ where and $\mathbf{V}_{\mathbf{R}}$ the relative velocity between the contacting particles, $\mathrm{K}_{\mathrm{n}}=\frac{\mathrm{m}_{\text {eff }}}{\mathrm{t}_{\text {contact }}} \ln (\epsilon)^{2}+\pi^{2}$ is the spring stiffness, $\mathrm{C}_{\mathrm{n}}=\frac{2 \ln (\epsilon) \sqrt{\mathrm{K}_{\mathrm{n}} \mathrm{m}_{\text {eff }}}}{\sqrt{\ln (\epsilon)^{2}+\pi^{2}}}$ is the viscous damping coefficient, $\overline{\mathbf{n}}$ the normal at contact, $\epsilon$ is the coefficient of restitution and $\mathrm{m}_{\mathrm{eff}}=\left(\frac{1}{\mathrm{~m}_{1}}+\frac{1}{\mathrm{~m}_{2}}\right)^{-1}$ is the effective mass of the particles. The contact time $t_{\text {contact }}$ is determined by the properties of the material and is chosen such that physical quantities of interest (such as energy) are conserved during integration for the typical range of velocities observed in the simulations [31, 18, 32]. For all simulations in this study we use a contact time equivalent to at-least 10 time steps.

Tangent Contact Tangential contact between particles are resolved using the Cundall Stark tangential model and is coupled to the normal force through Coulomb's law. The initial tangential force is computed as the sum of the tangential spring force and a tangential viscous force

$$
\begin{gathered}
\mathbf{F}_{\mathrm{T}}^{\text {spring }}=-\mathrm{K}_{\mathrm{T}}\left(\mathbf{V}_{T} \mathrm{dt}\right)+\mathbf{F}_{\mathrm{T}}^{\prime}, \\
\mathbf{F}_{\mathrm{T}}^{\text {dash }}=\min \left(\mathrm{C}_{\mathrm{T}} \mathbf{V}_{\mathrm{T}}, \mu \mathbf{F}_{\mathrm{n}}^{\text {spring+dash }}\right),
\end{gathered}
$$

where $\mathbf{F}_{\mathrm{T}}^{\prime}$ is the previous tangent force projected onto the current tangential plane, $\mathrm{K}_{\mathrm{T}}$ the tangential spring stiffness and is typically set to be at least $\frac{1}{2} \mathrm{~K}_{n}$, and $\mathrm{C}_{\mathrm{T}}=\frac{2 \ln (\epsilon) \sqrt{\mathrm{K}_{\mathrm{T}} \mathrm{m}_{\text {eff }}}}{\sqrt{\ln (\epsilon)^{2}+\pi^{2}}}$ the tangential damping coefficient, and $\mathbf{V}_{\mathrm{T}}$ the relative tangential velocity. Note: We use the history dependent Cundall Stark model for the tangential force which is to the best of the authors knowledge the first time that this has been implemented on GPUs in an academic study.

Power Calculation The power drawn by a mill is largely determined by the dynamics of the charge within the mill thus we can obtain a good estimate of the power required by analyzing the energy loss mechanisms in a DEM simulation which is standard practice for such simulations. The total energy consumed by a mill is simply the net sum of the energy dissipated through contacts $E_{\text {diss }}=\sum_{i}^{K}\left(\left\|F^{\text {dash }}\right\| \triangle \mathrm{x}\right)$ where $\mathrm{K}$ is the number of contacts and $\Delta \mathrm{x}$ is the distance which the force acts. Here $\mathbf{F}^{\mathrm{d} a s h}$ is given by the disspative normal and tangential forces as defined in Equations 5 and 7 respectively. Thus the power consumed can be estimated by Power $=\frac{\mathrm{E}_{\text {diss }}}{\Delta \mathrm{t}}$ where $\Delta \mathrm{t}$ is the duration over which we wish to calculate the power.

\section{Experimental validation of GPU DEM}

The Blaze-DEM framework has been previously validated against experiment for hoppers with spheres and polyhedra[22] as well as spheres in ball mills[33]. In this paper we validate the volume based contact method for polyhedra in ball mills as well as bi-disperse spherical particles using the incremental spring tangent model that was absent in previous works owing to the challenges of efficiently implementing contact history on the GPU. 


\subsection{Setup}

To validate our code we used a laboratory scale slice mill having a diameter of $90 \mathrm{~cm}$ and length of $15 \mathrm{~cm}$ with eight steel square or wooden trapezoidal lifters as depicted in Figure 3.1. The media in the mill was either steel balls $\left(2,1.5,0.50,0.25\right.$ inches) with a density of $7850 \mathrm{~kg} \cdot \mathrm{m}^{-3}$ or 1 inch aluminum cubes having a density of $2800 \mathrm{~kg} \cdot \mathrm{m}^{-3}$. Furthermore the mill is fitted with a torque sensor to provide power information and Plexiglas front to capture the charge motion.

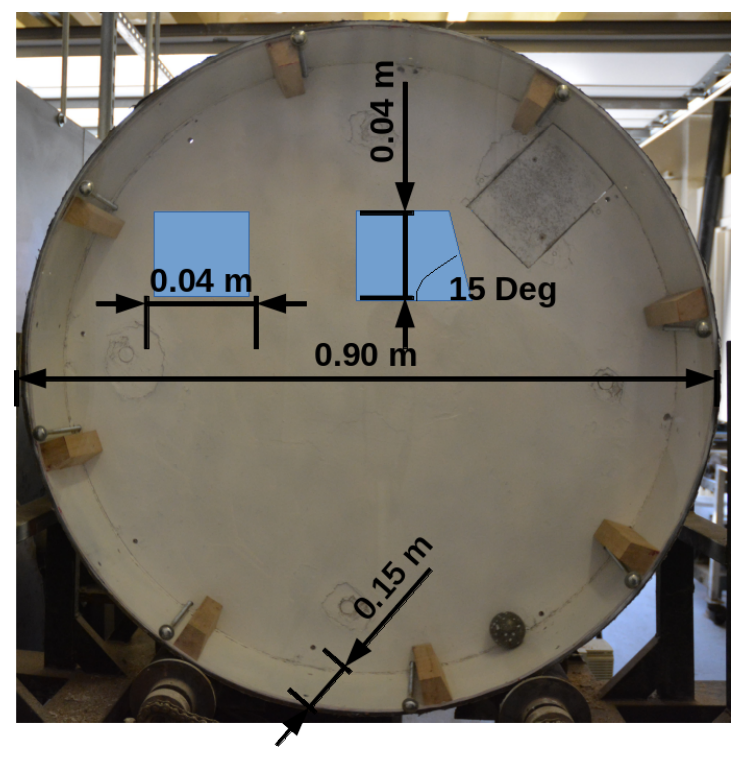

Figure 6: Experimental setup .

\subsection{Validation for polyhedral particles}

Table 1 contains the parameters used for case 1 and 2 . We found that changing the stiffness parameter and time step value has little effect on the charge profile which is inline with other studies provided the spring stiffness and time step are numerically stable. Thus in addition to a maximum relative overlap of $2.5 \%$ we also require stiffness values that allow for at least 10 steps for a contact.

\begin{tabular}{llrr}
\hline Parameter & Symbol & Value & Unit \\
Normal Particle Stiffness & $\mathrm{K}_{\mathrm{N}}$ & $5 \times 10^{6}$ & $\mathrm{~N} / \mathrm{m}$ \\
Tangent Particle Stiffness & $\mathrm{K}_{\mathrm{T}}$ & $2.5 \times 10^{6}$ & $\mathrm{~N} / \mathrm{m}$ \\
Normal Damping & $\mathrm{C}_{\mathrm{N}}$ & 520 & \\
Tangent Damping & $\mathrm{C}_{\mathrm{T}}$ & 350 & \\
Restitution coefficient (PP) & & 0.60 & \\
Static friction (PP) & & 0.45 & \\
Kinetic friction (PP) & & 0.40 & \\
Restitution coefficient (PW) & & 0.5 & \\
Static friction (PW) & & 0.50 & \\
Kinetic friction (PW) & & 0.45 & \\
Explicit integration time step & $\Delta t$ & $1 \times 10^{-5}$ & $\mathrm{~s}$ \\
\hline
\end{tabular}

Table 1: Simulation parameters for DEM validation simulations of AL cubes, (PP) indicates particle-particle and $(\mathrm{PW})$ indicates particle-wall settings.

Case 1 : 12001 inch aluminum cubes, SQ Lifters 14 RPM Figure 7 (a) depicts the experiment at steady state with Figure 7 (b) depicting the DEM profile colored by velocity. We see a good match between the two with the shoulder, belly and toe positions in good agreement, the mean power with experiment was 
found to be $463 \mathrm{~W} \pm 9$ while the value predicted by DEM was $484 \mathrm{~W} \pm 7$ which is slightly higher due to the slightly higher belly position. It should also be noted that due to the additional degree of freedom from rotation we expect slight variations even at steady state.

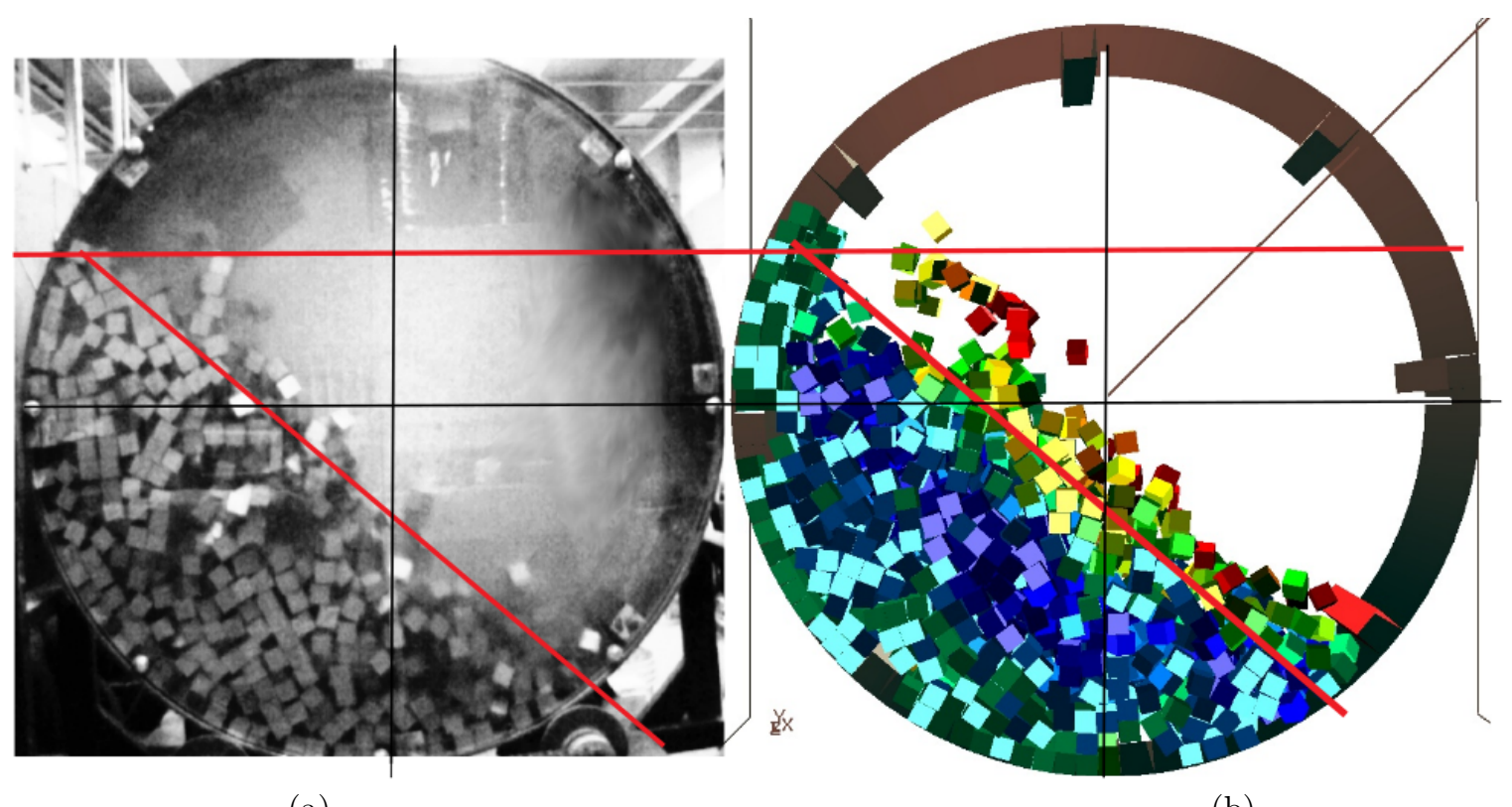

(a)

(b)

Figure 7: (a) Experimental and (b) DEM results at steady state for aluminum cubes at 14 RPM.

Case 2 : 12001 inch aluminum cubes, SQ Lifters 22 RPM We now increase the speed to 22 RPM to see if we can capture the change using the same parameters, we again see a good match between the two with DEM again have a slightly higher shoulder position. The mean power with experiment was found to be $761 \mathrm{~W} \pm 11$ while the value predicted by DEM was $781 \mathrm{~W} \pm 9$. 


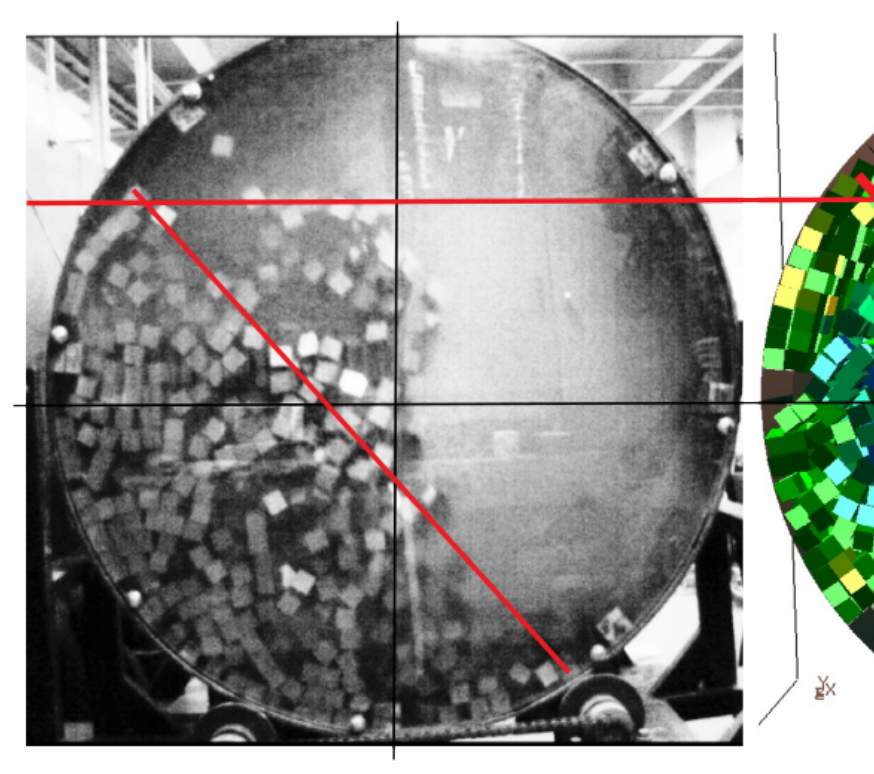

(a)

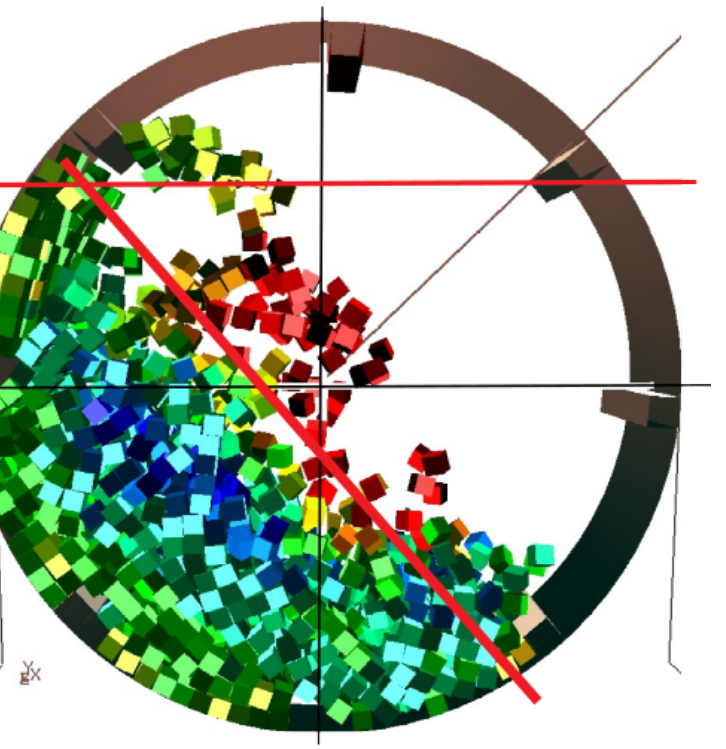

(b)

Figure 8: (a) Experimental and (b) DEM results at steady state for aluminum cubes at 22 RPM.

\subsection{Validation for spherical particles}

We now validate the code for spherical particles, in particular we do this for poly-disperse simulations which is a stronger of test of the code comparing the power draw and charge profiles at steady state. The parameters for the steel balls as well as the numerical only study in Section 4 is listed in Table 2.

\begin{tabular}{llrr}
\hline Parameter & Symbol & Value & Unit \\
Normal Particle Stiffness & $\mathrm{K}_{\mathrm{N}}$ & $9 \times 10^{6}$ & $\mathrm{~N} / \mathrm{m}$ \\
Tangent Particle Stiffness & $\mathrm{K}_{\mathrm{T}}$ & $4 \times 10^{6}$ & $\mathrm{~N} / \mathrm{m}$ \\
Normal Damping & $\mathrm{C}_{\mathrm{N}}$ & 155 & \\
Tangent Damping & $\mathrm{C}_{\mathrm{T}}$ & 100 & \\
Restitution coefficient (PP) & & 0.40 & \\
Static friction (PP) & & 0.55 & \\
Kinetic friction (PP) & & 0.50 & \\
Restitution coefficient (PW) & & 0.45 & \\
Static friction (PW) & & 0.55 & \\
Kinetic friction (PW) & & 0.50 & \\
Explicit integration time step & $\Delta t$ & $1 \times 10^{-5}$ & $\mathrm{~s}$ \\
\hline
\end{tabular}

Table 2: Simulation parameters for case 3-4 and Section 4 simulations, (PP) indicates particle-particle and (PW) indicates particle-wall settings.

Case 3 : 63 (2 inch) and 173 (1.5 inch) balls, 15 deg lifters 32 RPM. Figure shows the charge profile at steady state with a power draw of $551 \mathrm{~W} \pm 10$ and the predicted DEM value of $522 \mathrm{~W} \pm 15$. 


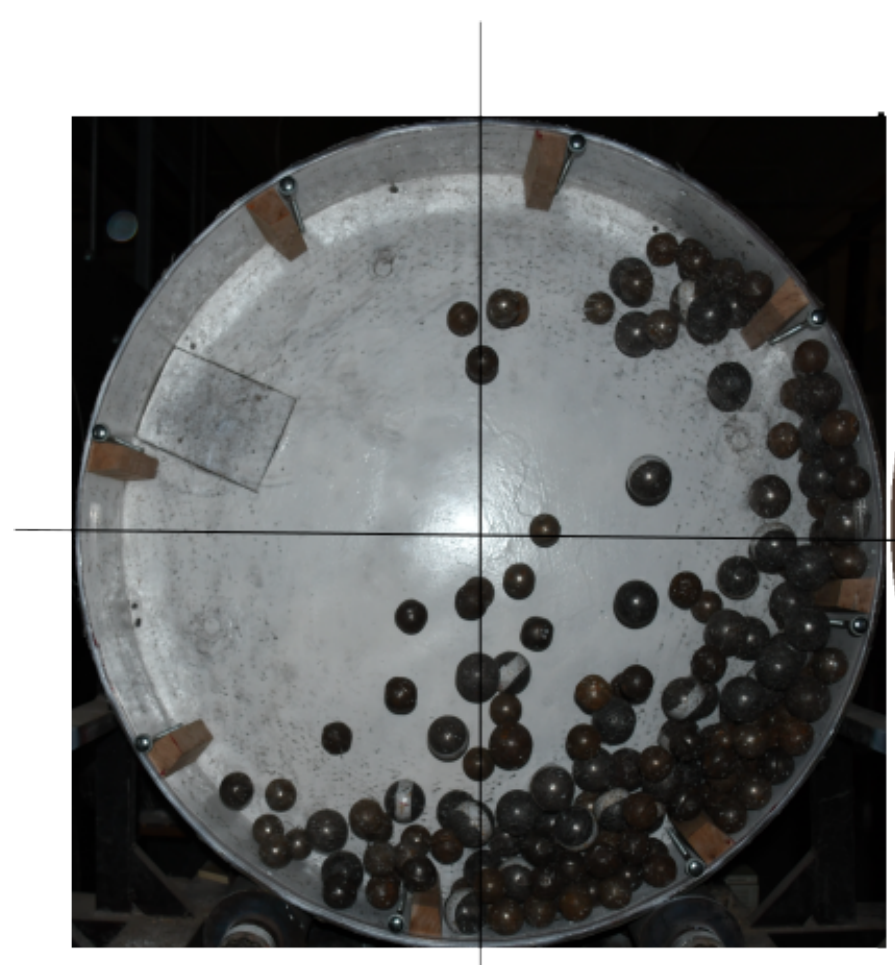

(a)

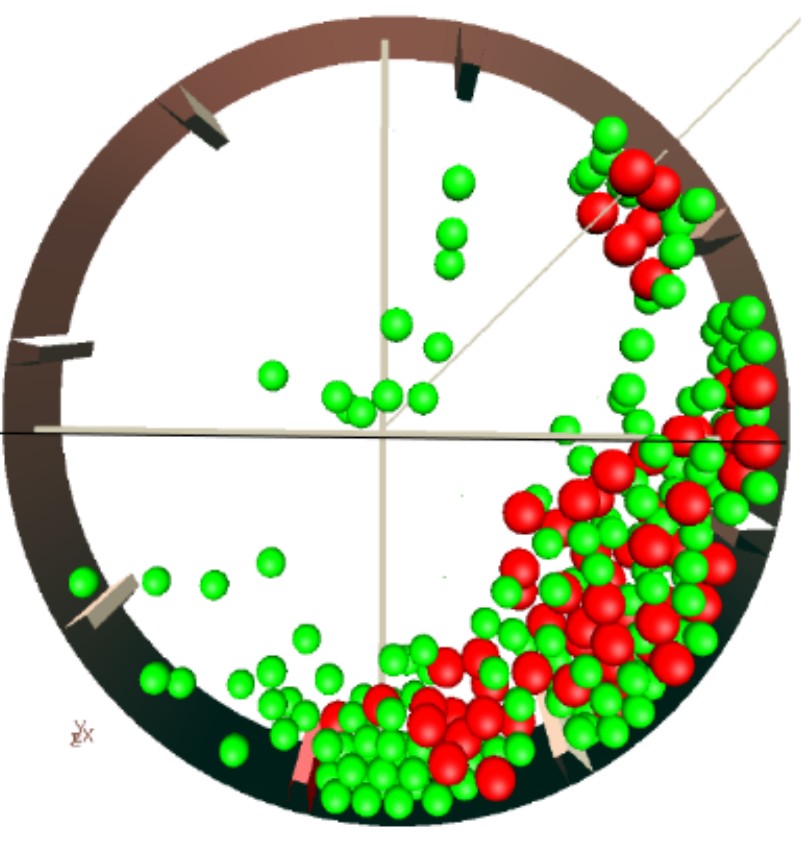

(b)

Figure 9: (a) Experimental and (b) DEM results at steady state for 2 inch and 1.5 inch balls angled lifter.

Case $4: 63$ (2 inch) and 173 (1.5 inch) balls, SQ lifters 32 RPM. We now remove the relief angle of the lifter to see if we can predict the correct dynamics with DEM. Figure shows the charge profile at steady state with a power draw of $631 \mathrm{~W} \pm 13$ and the predicted DEM value of $618 \mathrm{~W} \pm 8$.

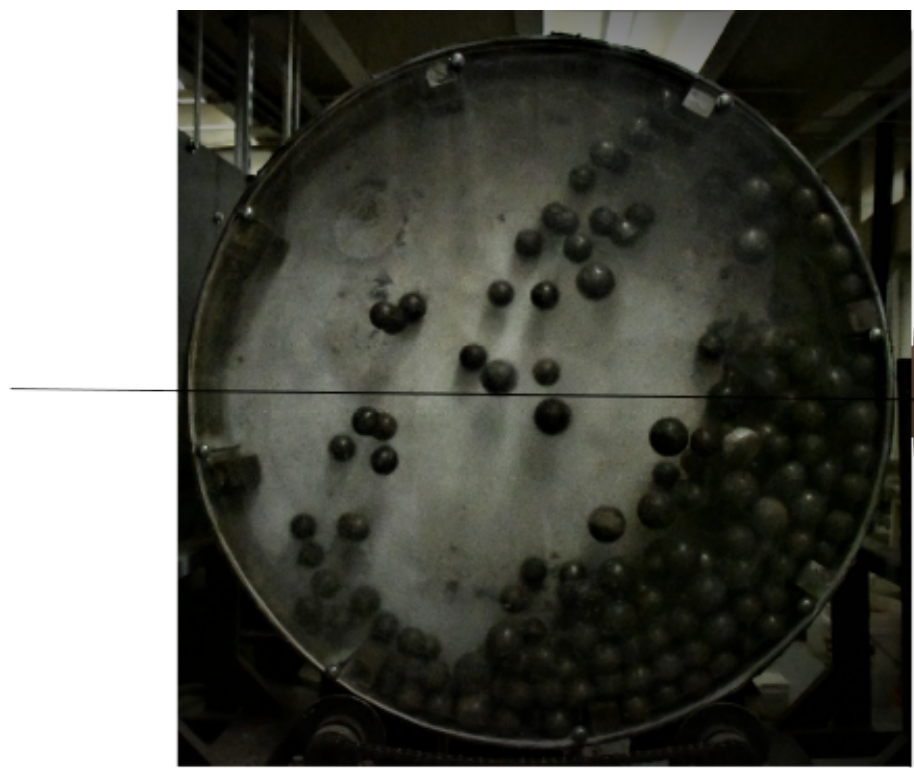

(a)

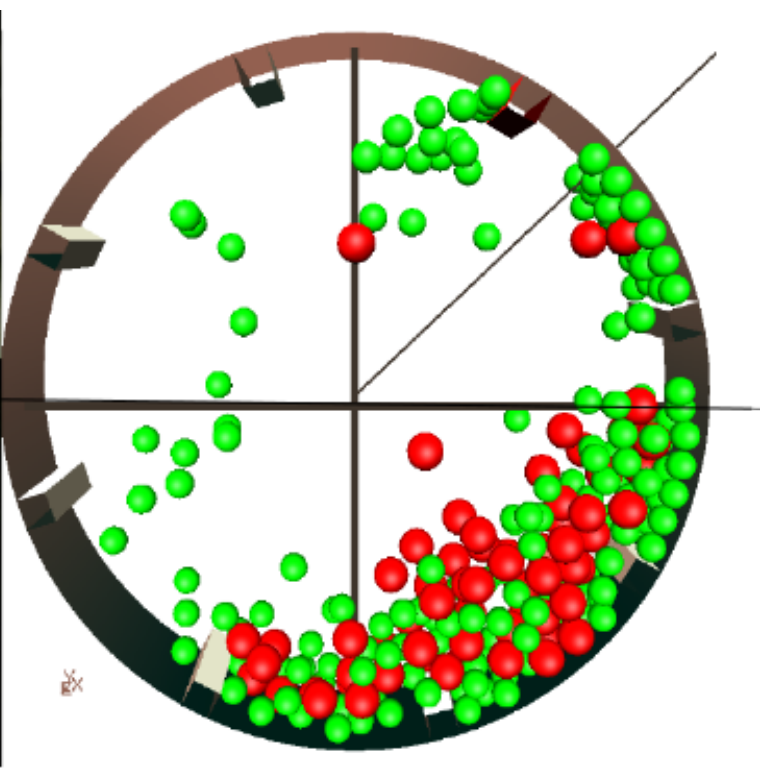

(b)

Figure 10: (a) Experimental and (b) DEM results at steady state for 2 inch and 1.5 inch balls square lifter. 
Case 5: 3609 (0.5 inch) and 46508 (0.25 inch) balls, 15 Deg Lifters 32 RPM We now decrease the size of the particle so than we can get a better understanding on the ability of the code to predict macroscopic behavior. The power draw from experiment is $685 \mathrm{~W} \pm 6$ while the predicted from DEM is $704 \mathrm{~W}$. This is due to DEM having a slightly lower shoulder resulting in a lower belly and higher toe position than experiment. However both the power draw and charge profile are well within acceptable values.

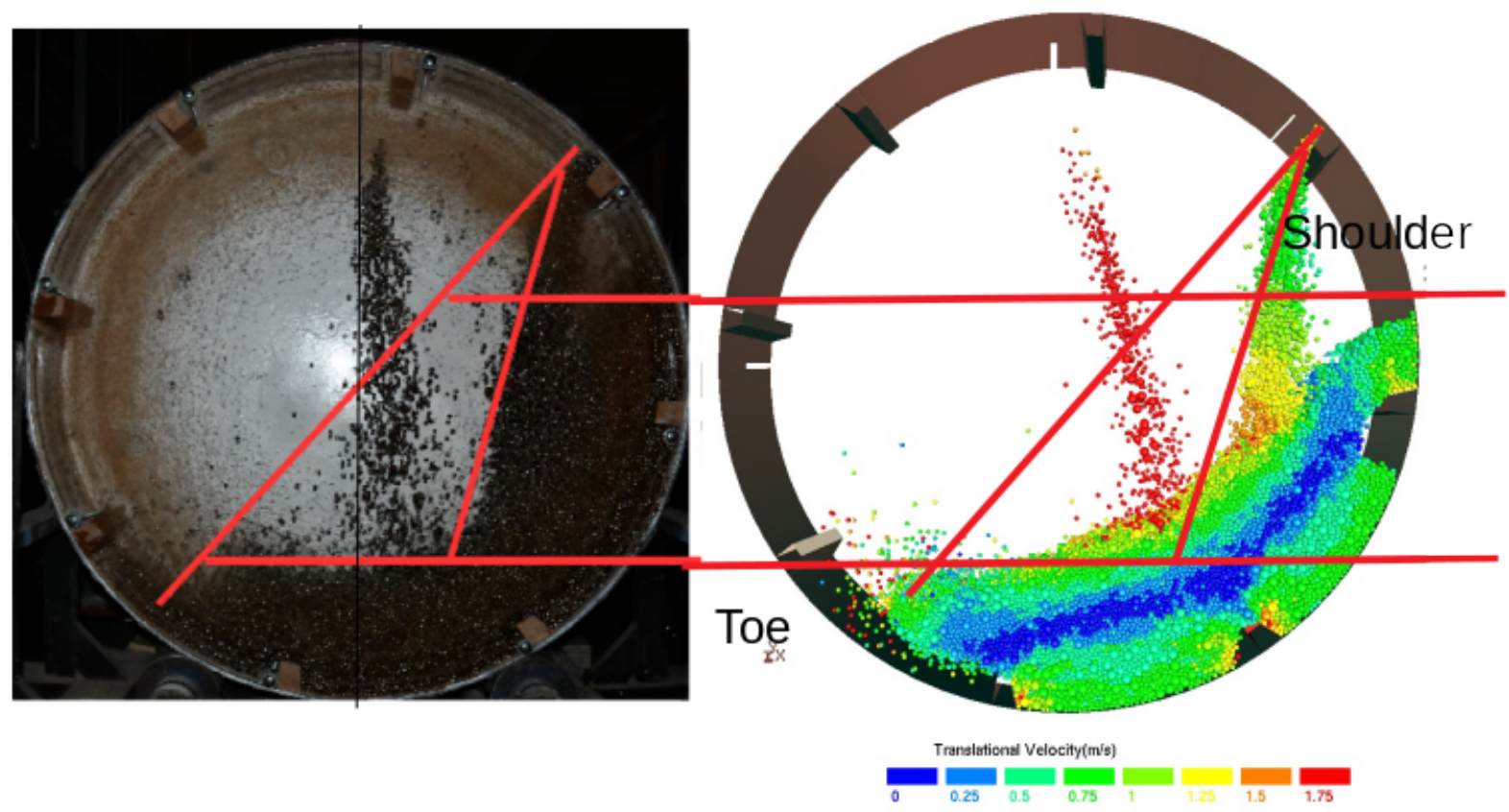

(a)

(b)

Figure 11: (a) Experimental and (b) DEM results at steady state for 0.50 inch and 0.25 inch balls.

\section{Effect of Particle Shape}

Now that we have validated our contact method for polyhedra we gauge the effect of particle shape on power draw and charge profile. The particles considered in this study are depicted in Figure 4, where particles have increasing angularity, symmetry and aspect ratios keeping the volume constant. Polyhedral shaped particles are modeled with full angularity as opposed to the clumped sphere and superquadric approximations. Table 3 lists the geometric and mass properties for the various particles used including bounding radius, volume, aspect ratio, mass and surface area. The aspect ratios (maximum and minimum extend in any direction) of the particles varied between 1 and 6.68, while all the particles had similar masses within $3 \%$ giving a total mass of approximately $47 \mathrm{~kg}$ per particle system. The drum rotated at $32 \mathrm{RPM}$ and was simulated up to $15 \mathrm{~s}$ of simulation time.

Computational Performance One of the biggest challenges of the DEM is the computational run-time of simulations. However the GPU-based code Blaze-DEM that we use is one of the fastest codes for spherical particles and polyhedral particles[21, 22]. In this study the 15 s simulation of 32 thousand spheres took 5 minutes of a single GTX Titan X card while the polyhedra took 25 minutes on 4 GPUs in the slowest case (high aspect ratio hexagonal prism particle). In the case of these mill simulations the speed difference verses polyhedra is around 25, however it should be noted the number of contacts is much higher for the polyhedral particles which is which effects the computational time in addition to the number of particles. Our multi-gpu implementation is also highly efficient with an almost linear scaling to 4 GPUs for polyhedra. 


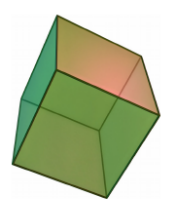

(a)

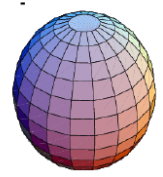

(b)

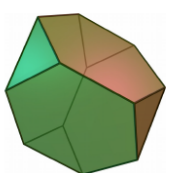

(c)

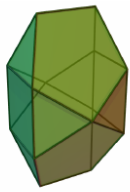

(d)

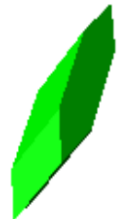

(e)

Figure 12: Particle shapes considered in this study include (a) cube, (b) sphere, (d) truncated tetrahedron (TTet), (d) bilunabirotunda (Biluna) and (e) scaled hexagonal prism (HexPrism)

\begin{tabular}{cccccc}
\hline Particle Shape & Bound Radius $\left(10^{-3} \mathbf{m}\right)$ & Volume $\left(10^{-6} m^{3}\right)$ & Aspect Ratio & Mass $\left(10^{-3} \mathbf{k g}\right)$ & SArea $\left(10^{-3} m^{2}\right)$ \\
Sphere & $\mathbf{1 . 0 0 0}$ & 0.523 & $\mathbf{1 . 0 0}$ & 4.12 & $\mathbf{3 . 1 4 1}$ \\
Cube & 0.732 & 0.523 & 1.00 & 4.26 & 3.89 \\
TTet & 0.714 & 0.528 & 1.15 & 4.14 & 4.072 \\
Biluna & 0.767 & 0.523 & 1.48 & 4.14 & 3.727 \\
HexPrism & $\mathbf{1 . 2 0 0}$ & 0.524 & $\mathbf{6 . 6 8}$ & 4.12 & $\mathbf{5 . 1 7 2}$ \\
\hline
\end{tabular}

Table 3: Particle geometric properties

Velocity Profile We now consider the steady state solution after 4 revolutions as depicted in Figures4(a)(e). The Biluna and HexPrism now have the largest dead zones. In turn the Cube and TTet have similarly defined dead zones, while the Sphere particle system has the least defined dead zone. The implication is that the abrasion characteristics between particle systems will differs significantly. It is evident that the Cube particles still mainly impact after the bulk toe with the mill shell, whilst the other particle systems mainly impact before the bulk toe on the particle cascade. Consider the particles feeding from the lifter into the cascading zone, it is clear that the Biluna and HexPrism particle systems are closely related, while the TTet particle system has a slightly higher build up against the mill wall. The build up from the cascade region to the mill wall for the Sphere and Cube particle systems are significantly higher due to the regular packing of the particles. The feed slope for the Sphere particle system is also much steeper due to the ordered packing collapsing from the wall onto the cascade, while the Cube particle system tends to interlock before collapsing. 


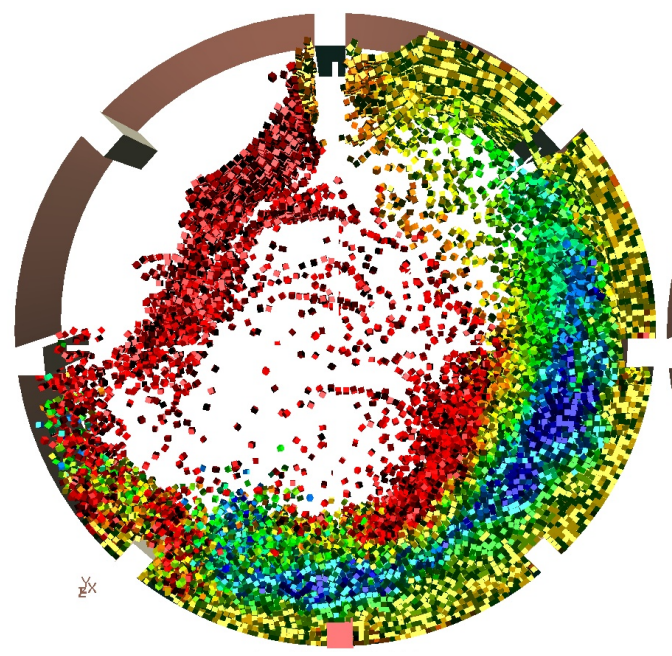

(a)

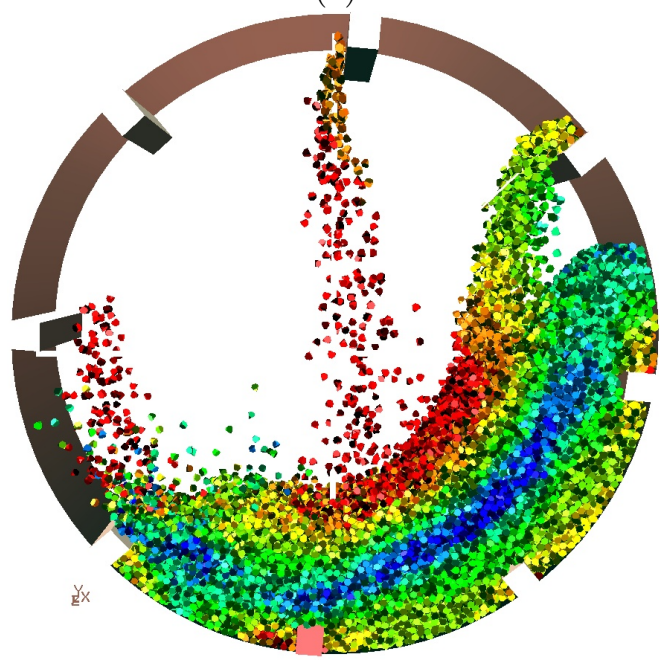

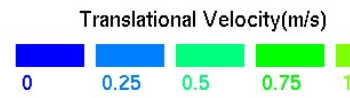

(c)

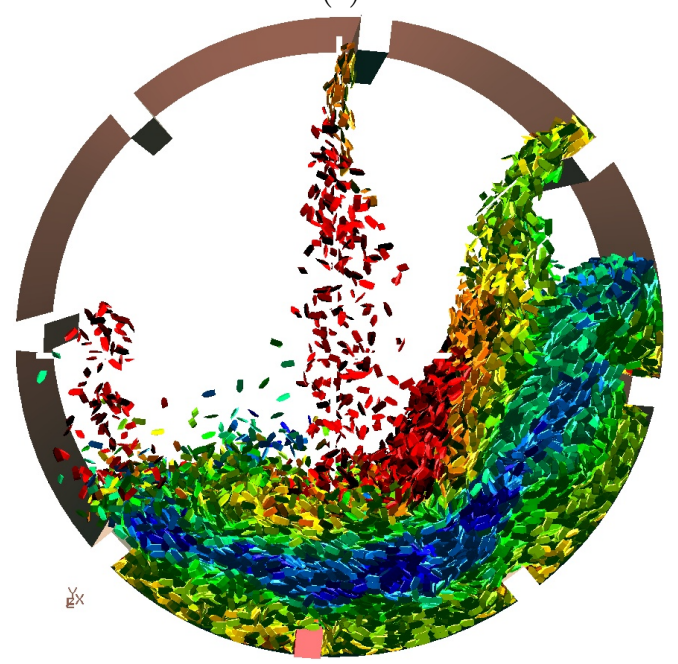

(e)

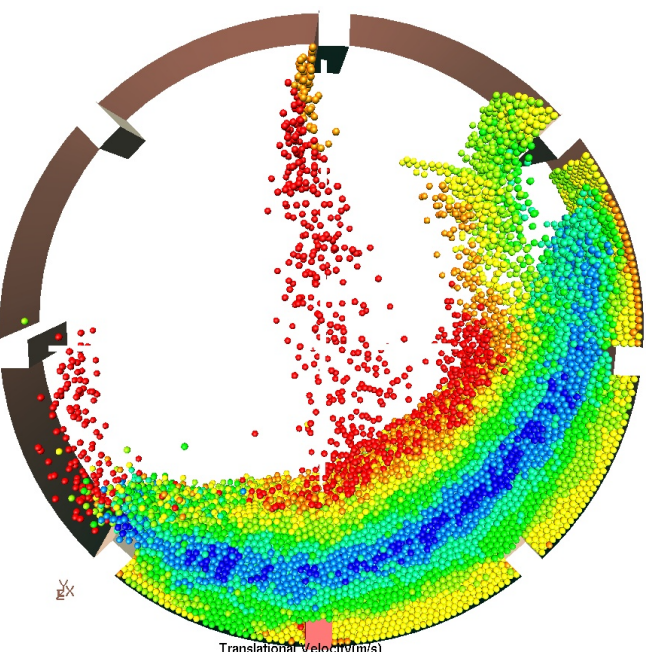

(b)

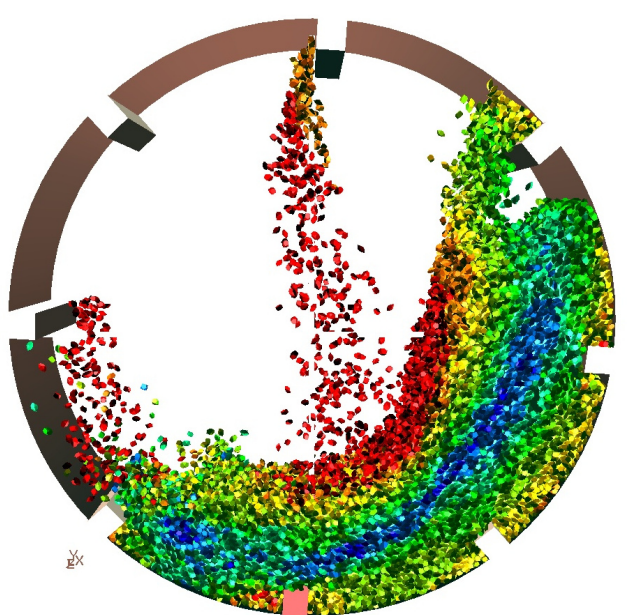


Overall Power The power draw and dissipation characteristics for the various particle systems are given in Table4. The total power draw as well as the power dissipation profile for different interaction modes are listed in Table 4. These include normal interactions between particles (PartN), normal interactions between particles and lifter (LiftN) and the mill shell, shear between particles (PartS), shear between particles. Note all power values are the average of 5 simulations for each case where the initial packing of the polyhedra was varied, we only noticed a variation of $1.2 \%$ in the most extreme case at steady state. We omitted the lifter shear and shell shear as the values where in all cases $<10 \mathrm{~W}$.

The largest total power is drawn by the Cube particle system, which in the context of the considered velocities profiles makes sense. For the Cube particle system the bulk of the particles is lifted much higher, in addition to particles interacting the mill shell behind the toe of the particle cascade. The TTet, HexPrism and Mix particle systems have similar total power draw requirements (variations of $15 \mathrm{~W}$ ) and required $14 \%$ less than the Cube particle system. In turn, the Sphere particle system had the lowest power requirements and required $27 \%$ less total power draw than the Cube particle system. The Biluna particle system required the least total power draw of all the polyhedral particle systems, which is $21 \%$ less than the Cube particle system. The Biluna particle system is also the least angular (most spherical) polyhedral particle system resulting in a lower power draw. Additional insight into the power dissipation of the particle systems can be gained by considering the shear and normal power contributions.

Normal and Shear Power Listed in Table4 are the normal and shear particle-particle (PartN and PartS) dissipation's, normal particle-lifter (LiftN) dissipation and the normal particle-shell dissipation (ShellN). The Cube particle system has the highest normal particle-particle dissipation's as well as the the highest normal particle-shell dissipation. The normal particle-particle dissipation for the Cube system is three times more than the Sphere particle system, in fact, the Cube particle system has very little shear dissipation between particles less than half of any other particle system. In turn, the shear dissipation between particles is dominated by the Sphere particle system, which is $34 \%$ more than the particle system with the second highest shear dissipation between particles, namely the TTet particle system. The normal impacts with the shell is dominated by the Cube particle system as the particles impact behind the toe of the particle cascade, the Cube particle system dissipation for particle-lifter interactions is $35 \%$ more than the second highest dissipation namely the Biluna particle system. Of the polyhedral particle systems the Biluna has the lowest normal power dissipation between particles, which is as a result of the lower angularity of this particle w.r.t. to the other particles resulting in a particle cascade that is more reminiscent to rolling than tumbling. The Sphere particle system dissipates the most power w.r.t. the particle-lifter interactions.

\begin{tabular}{cccccc}
\hline Particle Shape \Units (W) & Total Power & PartN & PartS & LiftN & ShellN \\
Sphere & 305 & 98 & $\mathbf{1 1 7}$ & $\mathbf{6 3}$ & 26 \\
Cube & $\mathbf{4 2 0}$ & $\mathbf{2 8 4}$ & 31 & 33 & $\mathbf{6 3}$ \\
TTet & 375 & 214 & 87 & 42 & 27 \\
Biluna & 332 & 169 & 66 & 53 & 38 \\
HexPrism & 360 & 216 & 68 & 44 & 29 \\
Mix & 350 & 200 & 70 & 41 & 32 \\
\hline
\end{tabular}

Table 4: Power dissipation for the various particle systems after 3.75 seconds for one revolution.

An alternative quantification to the total power is to consider the percentage power of the total power that is dissipated by the various modes of dissipation as listed in Table 4 . It is evident that the Cube, TTet, HexPrism and Mix particle systems dissipate at least $57 \%$ of their total power as normal interactions between particles, while the Biluna and Sphere particle systems dissipate less than $51 \%$ of their power in that mode. For the Cube system shear interactions dissipated only $7 \%$ of the total power, while for the other particle systems shear interactions contributed between $19 \%$ and $38 \%$ of the total dissipated power. The Sphere particle system dissipates $21 \%$ of its power w.r.t. the particle-lifter interactions, which becomes evident when exploring the particle lifter interactions w.r.t the force chain network. 


\begin{tabular}{ccccc}
\hline Particle Shape $\backslash$ Units (\%) & \% PartN & \% PartS & \% LiftN & \% ShellN \\
Sphere & 32 & $\mathbf{3 8}$ & $\mathbf{2 1}$ & 9 \\
Cube & $\mathbf{6 8}$ & 7 & 8 & $\mathbf{1 5}$ \\
TTet & 57 & 23 & 11 & 7 \\
Biluna & 51 & 20 & 16 & 11 \\
HexPrism & 60 & 19 & 12 & 8 \\
Mix & 57 & 20 & 12 & 9 \\
\hline
\end{tabular}

Table 5: Percentage power dissipation w.r.t. total power dissipation for the various particle systems after 3.75 seconds for one revolution.

Force Chain Network Consider the Sphere and Cube force chain networks respectively depicted in Figures 4(a)-(b) and Figures 4(c)-(d). The front and bottom side (five o'clock) views are shown. It is evident that both the Sphere and Cube particle system result in regular ordered packing's due to the symmetries of these two particle systems. In adaptation, the forces between the sphere and the lifters are the largest for the sphere particle system due to the force network and more uniform for the Cube particle system. The particle cascade for the Sphere has a fuller belly than the Cube particle system, which is also evident from the velocity profiles. As a result of this the Sphere particle system has a much denser network of force chains. 


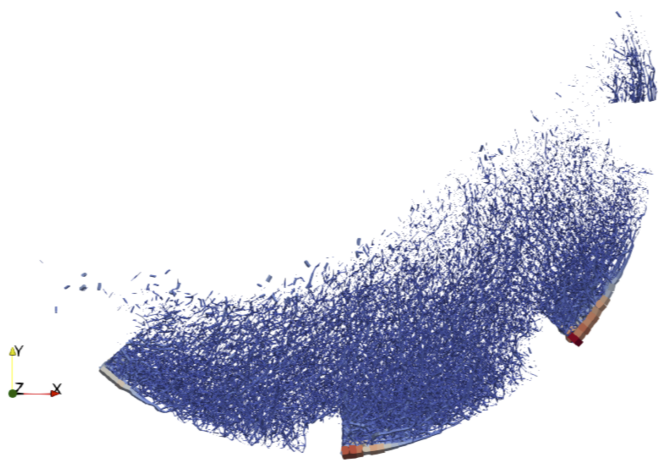

(a)

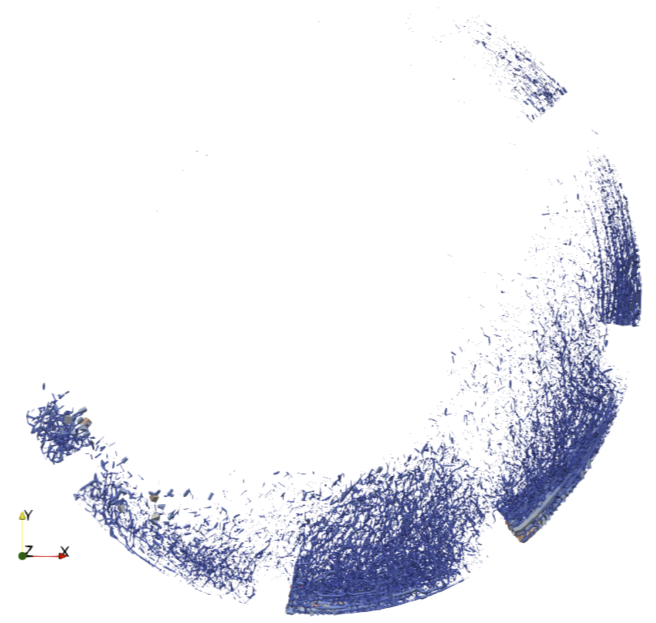

(c)

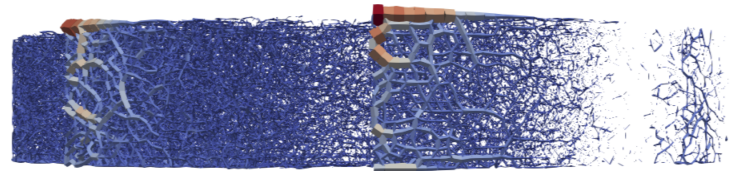

(b)
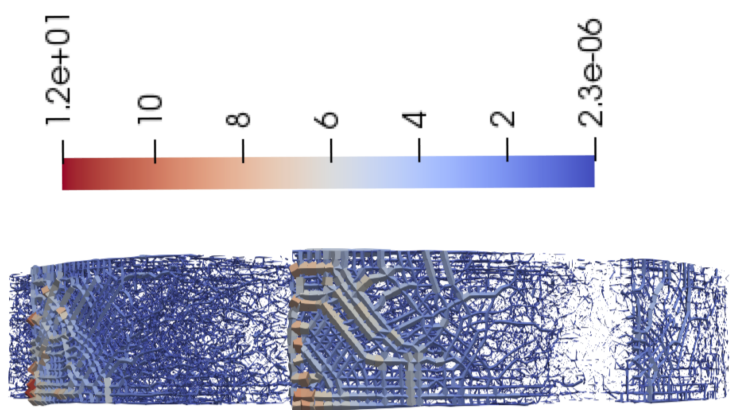

(d)

Figure 14: The front and bottom side (five o'clock) views of the force chain networks of of the (a)-(b) Sphere and (c)-(d) Cube particle systems.

The associated force chain network for the various polyhedral particle systems are depicted in Figures 4(a)(j) from the side and bottom five o'clock vantage points. The well developed and ordered force chain network for the Cube particle system is evident from the bottom five o'clock view as depicted in 4(b). Similarly, but with lower intensity is the force network for the Biluna particle system depicted in 4 (f). Additionally, from the side view it is evident that the Cube particle system has a much wider developed force chain network extending from just below 9 o'clock to 1 o'clock, while the other networks extend from 7 o'clock to 2 o'clock. This is also why the cascade for the Cube network is smaller than the other networks. Also, it is evident that the TTet network is the densest throughout the cascade. Differences in force networks evidently result in differences in the number of contacts as well as the the energies associated with these contacts. 


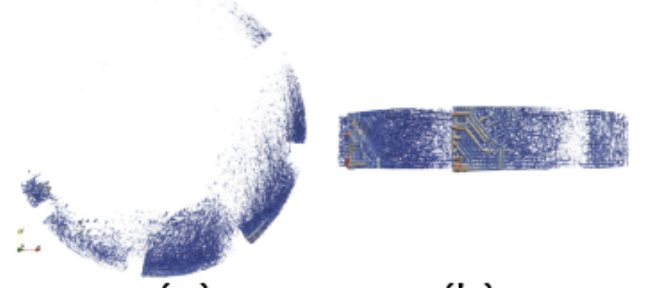

(a)

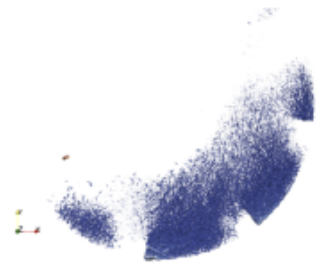

(e)

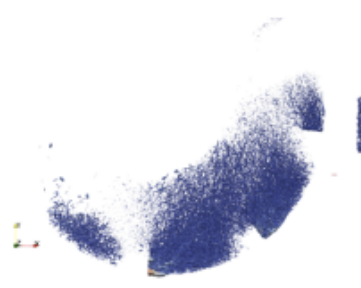

(i) (b)

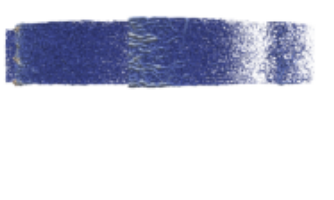

(f)

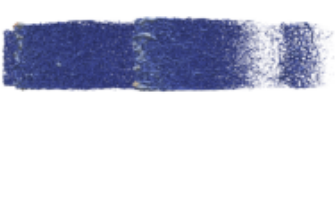

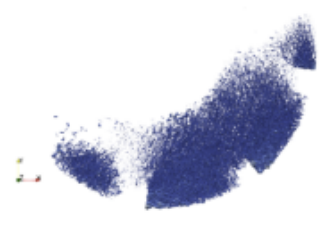

(c)

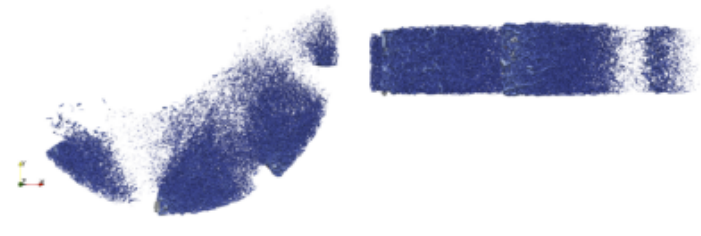

(g) (d)

(h)

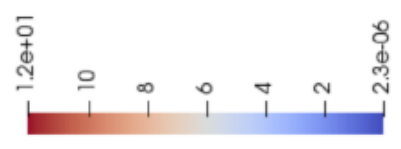

Figure 15: The front and bottom side (five o'clock) views of the force chain networks of of the (a)-(b) Cube, (c)-(d) TTet, (e)-(f) Biluna, (g)-(h) HexPrism and (i)-(j) Mix particle systems.

Normal and Shear Energy Spectra Lastly we therefore quantify the differences between the various particle systems is to plot the number of contacts for different normal and shear energies, respectively denoted normal and shear energy spectra, as depicted in Figures 4(a) and (b). For both plots, the number of contacts are normalized with respect to the Sphere particle system. At high normal energies all polyhedral (Cube, TTet, Biluna HexPrism) and the Mix particle systems have between two and four times more number of contacts than the Sphere particle system. This is evident from the tumbling nature of particles in polyhedral particle systems with respect to the rolling nature of spheres in the Sphere particle systems. In addition, the Biluna particle system has its lowest number of contacts associated with the lowest normal energy, while the TTet particle system has its highest energy associated with the highest normal energy. For intermediate energies all particle systems have more contacts than the Sphere particle systems except the Hex and Biluna particle systems. At high shear energies the TTet, Biluna, HexPrism and Mix particle systems have more number of contacts than the Sphere particle system. The Cube particle system has on average around the same number of contacts than the Sphere particle system. The shear energy spectra for the TTet, Biluna and Mix particle systems are very similar, while the Hex and Cube particle systems have distinct shear energy spectra. The TTet particle system has the largest shear energy spectra. 

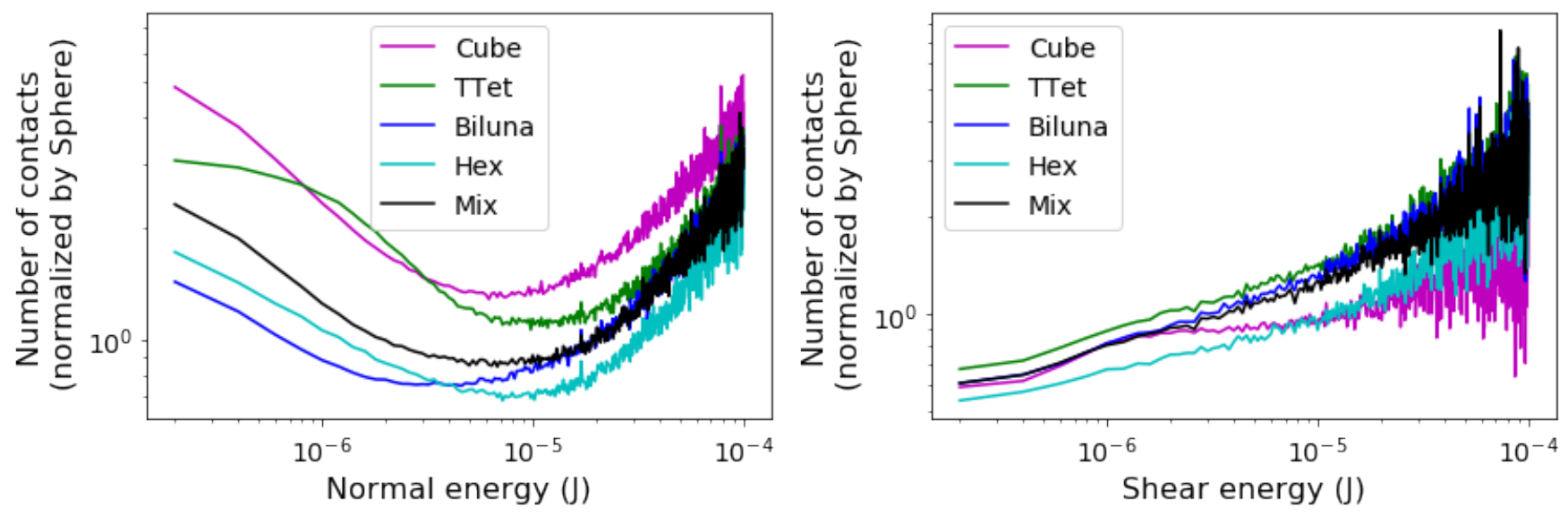

Figure 16: The number of contacts around different (a) normal and (b) shear energies, respectively denoted normal and shear energy spectra, for the various particle shapes considered in this study, which include Cube, TTet, Biluna, HexPrism and Mix. For both plots, the number of contacts are normalized with respect to the Sphere particle system.

\section{Conclusion}

The influence of particle shape on total power draw, power dissipation, energy spectra and velocity profiles have been demonstrated. Particle shape can result in $25 \%$ difference in total power draw for a ball mill / tumbling mill operation. The Cube particle systems dissipate around $420 \mathrm{~W}$, while the Sphere particle system dissipates around $300 \mathrm{~W}$. Significant differences in power dissipation mechanisms influenced by particle shape was also highlighted e.g. Cube, TTet, HexPrism and Mix particle systems dissipate at least $57 \%$ of their total power as normal interactions between particles, while the Biluna and Sphere particle systems dissipate less than $51 \%$ of their power in that mode. For the Cube system shear interactions dissipated only $7 \%$ of the total power, while for the Sphere, TTet, Biluna, HexPrism and Mix particle systems the shear interactions contributed between $19 \%$ and $38 \%$ of the total power dissipated. This study again highlights the importance of considering accurate representations of particle shape in comminution applications.

\section{Acknowledgments}

This work was supported by the MARIE Sklodowska-CURIE Individual Fellowships with acronym DECRON, funded through the People Programme (MARIE Sklodowska-CURIE Actions) of the European Union's H2020 under REA grant agreement No. 747963. We gratefully acknowledge the support of the NVIDIA Corporation with the donation of the Titan X Pascal GPU used for this research. The financial support of the National Research Foundation (NRF) of South Africa is acknowledged. 


\section{References}

[1] P. W. Cleary. Recent advances in DEM modelling of tumbling mills. Minerals Engineering, 14:1295-1319, 2001.

[2] Paul W. Cleary, Rob Morrisson, and Steve Morrell. Comparison of DEM and experiment for a scale model SAG mill. International Journal of Mineral Processing, 68:129-165, 2003.

[3] R.D. Morrison and Paul W. Cleary. Using DEM to model ore breakage within a pilot scale SAG mill. Minerals Engineering, 17:1117-1124, 2004.

[4] Paul W. Cleary and R.D. Morrison. Comminution mechanisms, particle shape evolution and collision energy partitioning in tumbling mills. Minerals Engineering, 86:75-95, 2016.

[5] Raj K. Rajamani, Samira Rashidi, and Nikhil Dhawan. Advances in discrete element method application to grinding mills, pages 117-128. Society for Mining, Metallurgy and Exploration (SME), 2014.

[6] R.K Rajamani, S. Callahan, and J. Schreiner. DEM Simulation of mill charge in 3D via GPU computing. Proceeding of the SAG conference, Vancouver, 2011.

[7] Yuxing Peng, Tongqing Li, Zhencai Zhu, Shengyong Zou, and Zixin Yin. Discrete element method simulations of load behavior with mono-sized iron ore particles in a ball mill. Advances in Mechanical Engineering, 9(5):1687814017705597, 2017.

[8] Parviz Pourghahramani. Effects of ore characteristics on product shape properties and breakage mechanisms in industrial SAG mills. Minerals Engineering, 32:30-37, 2012.

[9] Shigeto and Sakai. Parallel computing of discrete element method on multi-core processors. Particuology, 9:389-405, 2011.

[10] D. Zhao, E.G. Nezami, Y. Hashash, and Jamshid Ghaboussi.J. Three-dimensional discrete element simulation for granular materials. Computer-Aided Engineering Computations: International Journal for Engineering and Software, 23:749-770, 2006.

[11] Seung Jaelee. PhD Thesis:developments in large scale discrete element with polyhedral particles simulations. University of Illinois at Urbana-Champaign,, www.uiuc.edu, 2014.

[12] S. Mack, P. Langston, C. Webb, and York.T. Experimental validation of polyhedral discrete element model. Powder Technology, 214:431-442, 2011.

[13] N. Govender, D. Wilke, and S. Kok. A GPU based polyhedral particle DEM transport code. NVIDIA GTC 2014, San Jose,USA, March 2014.

[14] C.A. Radeke, B.J. Glasser, and J.G. Khinast. Large-scale powder mixer simulations using massively parallel GPU architectures. Chemical Engineering Science, 65:6435-6442, 2010.

[15] N. Govender, D. Wilke, S. Kok, and R. Els. Development of a convex polyhedral discrete element simulation framework for NVIDIA Kepler based GPUs. JCAM, 270:63-77, 2014.

[16] Zhang and et.al. A fast scalable implementation of the two-dimensional triangular Discrete Element Method on the GPU platform. Advances in Engineering Software, 60:70-80, 2013.

[17] J. Xu and et.al. Quasi-real-time simulation of rotating drum using discrete element method with parallel GPU computing. Particuology, 9:446-450, 2011.

[18] Marius Hromnik. Masters Thesis: A GPGPU implementation of the discrete element method applied to modeling the dynamic particulate environment inside a tumbling mill. University of Cape Town, www.uct.ac.za, 2013.

[19] J.P Longmore, P. Marais, and M. Kuttel. Towards realistic and interactive sand simulation: A GPUbased framework. Powder Technology, 235:983-1000, 2013.

[20] H. Abou-Chakra, J. Baxter, and U. Tuzun. Three-dimensional particle shape descriptors for computer simulation of non-spherical particulate assemblies. Advanced Powder Technology, 15:63-77, 2004. 
[21] N. Govender, D. Wilke, and S. Kok. Collision detection of convex polyhedra on the NVIDIA GPU architecture for the discrete element method. Journal of Applied Mathematics and Computation, http://dx.doi.org/10.1016/j.amc.2014.10.013, 2014.

[22] Nicolin Govender, Daniel N. Wilke, Patrick Pizette, and Nor-Edine Abriak. A study of shape non-uniformity and poly-dispersity in hopper discharge of spherical and polyhedral particle systems using the blaze-dem gpu code. Applied Mathematics and Computation, http://dx.doi.org/10.1016/j.amc.2017.03.037:318-336, 2017.

[23] B. Smeets, T. Odenthal, S. Vanmaercke, and H. Ramon. Polygon-based contact description for modeling arbitrary polyhedra in the Discrete Element Method. Computer Methods in Applied Mechanics and Engineering, 290:277-289, jun 2015.

[24] Victor Klee. Maximal Separation Theorems for Convex Sets. Transactions of the American Mathematical Society, 134(1):133-147, 1968.

[25] Helge Tverberg. A separation property of plane convex sets. Mathematica Scandinavica, 45(2):255-260, 1980 .

[26] C.W. Boon, G.T. Houlsby, and S. Utili. A new algorithm for contact detection between convex polygonal and polyhedral particles in the discrete element method. Computers and Geotechnics, 44:73-82, jun 2012.

[27] Jan Elias. DEM simulation of railway ballast using polyhedral elemental shapes. In III International Conference on Particle-based Methods Fundamentals and Applications, pages 1-10, 2013.

[28] Y T Feng, K Han, and D R J Owen. Energy-conserving contact interaction models for arbitrarily shaped discrete elements. Computer Methods in Applied Mechanics and Engineering, 205-208:169-177, 2012.

[29] D.E. Muller and F.P. Preparata. Finding the intersection of two convex polyhedra. Theoretical Computer Science, 7(2):217-236, jan 1978.

[30] F Tonon. Explicit Exact Formulas for the 3-D Tetrahedron Inertia Tensor in Terms of its Vertex Coordinates. Journal of Mathematics and Statistics, 1(1):8-11, 2004.

[31] R. Venugopal and R.K Rajamani. 3d simulation of charge motion in tumbling mills by the discrete element method. Powder Technology, 115:157-166, 2001.

[32] B.K. Mishra and R.K. Rajamani. Simulation of charge motion in ball mills. Part 1: experimental verifications. Int. J. Mineral Process, 40:171-186, 1994.

[33] N. Govender, R.K. Rajamani, D. Wilke, and S. Kok. Discrete element simulation of mill charge in 3d using the blaze-dem gpu framework. Journal of minerals engineering, http://dx.doi.org/10.1016/j.mineng.2015.05.010, 2015. 